\title{
PRESSURE AND RESPONSE IN COMPETITIVE INTERACTIONS
}

\author{
KENNETH MATHER* AND P. D. S. CALIGARI \\ * Department of Genetics, University of Birmingham, Birmingham B15 $2 T$ T. \\ Scottish Crop Research Institute (Pentlandfield), Roslin, EH25 9RF, U.K.
}

\section{SUMMARY}

In a group of organisms which are in competition with one another each individual plays a dual part: it will be exerting competitive pressure on its fellows and at the same time it will be responding to competitive pressure from them. The kind of competition experiment described by Mather and Caligari (1981) and Mather et al. (1982) allows differences in the pressure exerted by individuals of a given genotype (as the associate or aggressor) to be quantified separately from the response of that same genotype (as indicator or responder) to the pressure it experiences. Differences in pressure, or aggressiveness, and in response can be measured in terms of parameters $a$ and $r$ respectively and a value of each parameter, expressed as a deviation from the mean of the experiment, can be assigned to each genotype. The inter-relation of $a$ and $r$ can be measured by the interaction parameter $i$. The values of $a, r$ and $i$ are derived from the competition values $(c)$ of the various pairs of genotypes, which in turn are derived from the regressions of character expression on density of the indicator genotype in mono- and duo-cultures.

The analysis is applied to data from the Lolium experiment by Mather $e t$ $a l$., where the character is mean yield of dry matter per individual, and from four Drosophila experiments (T,L, A,C, C,H and X) using two characters, $p_{a}$ (the proportion of eggs developing successfully into adult flies) and $w$ (the mean weight of the adults so obtained). In two of the Drosophila experiments (A,C and $C, H) y^{2}$ was used as a marker gene and it is shown that of itself this gene had no significant effect on competitive ability. In a third experiment $(X)$ involving three genotypes, both $y^{2}$ and $w^{a}$ were used as markers.

In all three Drosophila experiments (T,L, A,C and C,H) where character $w$ was followed no difference is found in response as measured by $r$, in respect of this character, though in all cases differences are found in the pressure parameter, $a$. The same is true for character $p_{a}$ in experiments $T, L$ and $A, C$ but in the $\mathbf{X}$ experiment variation is observed among the three genotypes for both response and pressure. The $a$ and $r$ appear to be independent in their variation and they can be taken as combining additively in their contributions to the competitive value of the genotype. This experiment also suggests that there may be a relatively simple genetical basis for competitive ability as observed in it. In the remaining Drosophila experiment $(\mathrm{C}, \mathrm{H})$ significant values are found for both $a$ and $r$ but a strong interaction, $i$, between them is found also. This stems from the fact that one of the genotypes $(H, I)$, far from competing with the other $(C, D)$, facilitates its successful development into adults. C,D however still competes with $\mathrm{H}, \mathrm{I}$ (fig. 1).

In the Lolium experiment both $a$ and $r$ vary significantly among the three genotypes, in what appears to be a correlated fashion. They combine in a multiplicative rather than an additive way. The $a$ values vary more among the genotypes than do the $r$ values. The absolute values of $a$ and $r$ are dependent on the cutting regime, but the relative values of $r$ stay constant among the genotypes. The relative values of $a$ do, however, change with the regime.

The results are discussed in relation to the different mechanisms of competition which flies and grass plants must be expected to show. 


\section{INTRODUCTION}

In any group of organisms which are competing with one another, each individual plays a dual part in respect of the competitive interactions. On the one hand it will be exerting, at least prospectively, competitive pressure on all of its fellows in the group; and on the other it will be responding, at least prospectively, to the total competitive pressure exerted on it by its fellows. This distinction between pressure, or aggressiveness, and response, or sensitivity, was recognised by Breese and Hill (1973) in duocultures of weeds and of grass species. It was also inferred by Mather et al. (1982) from a competition experiment involving three genotypes of the perennial rye-grass, Lolium perenne. In the present report we are concerned with the separation of the two components of competitive interaction and with their properties in relation to each other.

\section{SOURCES OF DATA AND METHOD OF ANALYSIS}

The data that we shall use are provided by Mather et al.'s (1982) experiment with Lolium perenne and by four competition experiments with Drosophila melanogaster, the simplest of which is that described by Mather and Caligari (1981), where the two inbred lines Wellington (T) and 6CL (L) were in competition with one another.* In this experiment flies of the two types could be distinguished in mixed culture by their numbers of sternopleural chaetae, which were much higher in L than in T. In the other Drosophila experiments this ready means of distinction was not available as the lines used had overlapping distributions of this chaeta number. The introduction of a marker gene was thus necessary and $y^{2}$ was used for this purpose. It was introduced where necessary by single cross-overs from an $\mathrm{X}$ chromosome marked by $y^{2} w^{a} c v$ ct $v f$, the two large autosomes being held constant by the use of $\operatorname{In}(2 L R) P m a l^{4} d s^{33 k} l_{t} b w^{v i}$ and $\operatorname{In}(3 L R) D c x F ; D$ chromosomes. The success of $y^{2}$ as a marker will be examined when we come to discuss the results of these experiments in Section 4. $w^{a}$ was also used as a marker separate from $y^{2}$ in one experiment. This was introduced in the same way: $y^{2} w^{a}$ was first brought in as a unit and $y^{2}$ then removed by a further cross-over in a later generation.

Our method of analysis is an extension of that developed by Mather and Caligari (1981), and is most easily described by reference to their experiment with lines T and L of Drosophila. Their measure of the competitive interaction among flies of like genotype in a monoculture was the rate of change of the expression of the character under observation on the density of the culture as determined by the number of eggs with which it was started. The rate of change was measured as the slope of the regression line (denoted by $b_{m}$ ) relating expression to density, and represents the effect of reducing the competitive interaction by eliminating the contribution of one individual to the overall competition. If each individual of the primary (or indicator) genotype so omitted had been replaced by another of a different (secondary or associate) genotype, which however made an exactly equivalent contribution to the competitive pressure on the primary

\footnotetext{
* The amount of yeast added to each culture in this experiment is erroneously given as $6 \mathrm{mgm}$ in Mather and Caligari's paper. The correct amount is $60 \mathrm{mgm}$.
} 
genotype, the regression of the expression of the character shown by this primary genotype on its density in the culture would of course have been 0 , and the regression that it would have yielded is denoted by $b_{0}$. The intragenotype competitive interaction with themselves of the individuals of the primary or indicator genotype is thus measured by $b_{o}-b_{m}=-b_{m}$. Where, however, the omitted individuals of the primary genotype are replaced by an equal number of a secondary genotype having a different competitive effect, the regression of the expression of the character shown by the primaries on their density in the culture will not be 0 , and is estimated by $b_{d}$. The competitive value of the associate will thus be measured by $b_{d}-b_{m}$. The competitive values obtained by Mather et al., (1982) for the three genotypes of Lolium perenne were found in this way.

To revert to Mather and Caligari's experiment with the $\mathrm{T}$ and $\mathrm{L}$ inbreds of Drosophila, the value of $b_{m}$ obtained for T $\left(b_{m} r\right)$ and $\mathrm{L}\left(b_{m L}\right)$, and of $b_{d}$ obtained where $\mathrm{T}$ was treated as the indicator with $\mathrm{L}$ as associate $\left(b_{d T}\right)$ and where $\mathrm{L}$ was treated as indicator with $\mathrm{T}$ as associate $\left(b_{d L}\right)$ are repeated in table 1 for the two characters $p_{a}$ (proportion of eggs yielding adults, transformed into angles) and $w$ (mean weight of adult individuals). Denoting the response displayed by $T$ to its own competition as $c_{T T}$ and its response to competition from $\mathrm{L}$ as $c_{T L}$ we find $c_{T T}=-b_{m T}$ and $c_{T L}=b_{d T}-b_{m T}$.

TABLE 1

The values of $b_{m}$ and $b_{d}$ in the $T, L$ experiment with Drosophila

\begin{tabular}{ccrrrr}
\hline $\begin{array}{l}\text { Character } \\
\text { Indicator }\end{array}$ & $b_{m}$ & $p_{a}$ & \multicolumn{2}{c}{$w$} & \multicolumn{1}{c}{$b_{d}$} \\
\hline $\mathrm{T}$ & -0.353823 & -0.376145 & -0.0077887 & -0.0045030 \\
$\mathrm{~L}$ & -0.012477 & 0.142285 & -0.0027447 & 0.0073505 \\
\hline
\end{tabular}

Similarly, $c_{L L}=-b_{m L}$ and $c_{L T}=b_{d L}-b_{m L}$. The $c$ values so obtained are set out in table 2. The four $c$ values for each character have 3 degrees of freedom (df) among them and the overall sum of squares $(S S)$ can be partitioned into three items, each for $1 d f$, using orthogonal functions. On doing so for character $p_{a}$ we find

$$
\begin{aligned}
& R=\frac{1}{4}\left(c_{T T}+c_{T L}-c_{L T}-c_{L L}\right)^{2}=0.00674 \quad t_{12}=0.954 \quad p=0.4-0.3 \\
& A=\frac{1}{4}\left(c_{T T}-c_{T L}+c_{L T}-c_{L L}\right)^{2}=0.06719 \quad t_{12}=3.010 \quad p \simeq 0.01 \\
& I=\frac{1}{4}\left(c_{T T}-c_{T L}-c_{L T}+c_{L L}\right)^{2}=0.01367 \quad t_{12}=1.358 \quad p \simeq 0.2
\end{aligned}
$$

where $R$ tests the significance of the difference between $\mathrm{T}$ and $\mathrm{L}$ in their overall responses to the competition pressures exerted on them by the two of them as associates; $A$ tests the significance of the difference between $\mathrm{T}$ and $\mathrm{L}$ in the competitive pressures that they exert as associates; and $I$ tests for the interaction $(\boldsymbol{R} \times \boldsymbol{A})$ between competition pressure and response; (Note: Here we depart from the notation of Mather et al., who used $I$ for the item which we here denote by $R$ ).

The corresponding analysis in respect of character $w$ is:

$$
\begin{array}{ccl}
R=0.000000780 & t_{12}=0.842 & P=0.5-0.4 \\
A=0.000035129 & t_{12}=5.649 & P<0.001 \\
I=0.000002028 & t_{12}=1.357 & P=0.2
\end{array}
$$


TABLE 2

c values in the $T, L$ experiment with Drosophila Character $\mathrm{p}_{\mathbf{a}}$

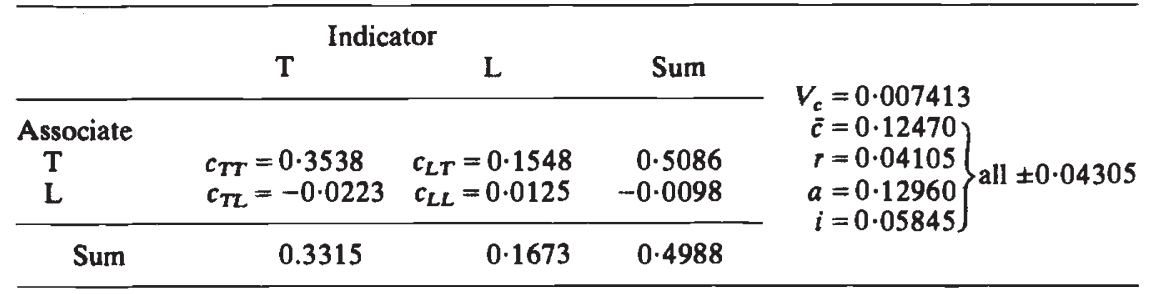

Character w

\begin{tabular}{|c|c|c|c|c|}
\hline \multicolumn{5}{|c|}{ Indicator } \\
\hline & $T$ & L & Sum & \multirow{5}{*}{$\left.\begin{array}{rl}V_{c} & =0.000001101 \\
\bar{c} & =0.0059790 \\
r & =-0.0004415 \\
a & =0.0029635\end{array}\right\}$ all \pm 0.0005} \\
\hline Associate & & & & \\
\hline & 0.007789 & 0.010096 & 0.017885 & \\
\hline L & 0.003286 & 0.002745 & 0.006031 & \\
\hline Sum & $0 \cdot 011075$ & 0.012841 & 0.023916 & \\
\hline
\end{tabular}

The error variance against which $R, A$ and $I$ are tested for each character, is derived from the duplicate error variance of the experiment in respect of that character. The values of these duplicate error variances are given by Mather and Caligari who also describe how the error variances of $b_{m}$ and $b_{d}-b_{m}$ are found from them. For character $p_{a}, V_{b m}=0.008063$ and $V_{(b d-b m)}=0.00673$. But $R, A$ and $I$ are each derived from two $b_{m}$ values and two $\left(d_{b}-d_{m}\right)$. The error variance against which they must be tested is thus $\frac{1}{2}(0.008063+0.006763)=0.007413$. The duplicate error variance from which these values are derived, was based on $12 \mathrm{df}$, and the test of significance thus becomes a $t_{12}$ in each case. The $t_{12}$ yielded by $R$, for example, is found as $\sqrt{0.00674 / 0.007413}=0.954$ with a probability falling between 0.4 and 0.3 . The error variance against which $R, A$ and $I$ must be tested for character $w$ was similarly found to be 0.000001101 .

The values of $t_{12}$ and their probabilities are shown above against the values of $R, A$ and $I$ for both characters. In both cases the two lines $\mathrm{T}$ and $L$ differ significantly in the competitive pressures that they exert whether on themselves in monoculture or on the other in duoculture; but in respect of neither character is there any good evidence that they differ in their response to the competitive pressure exerted on them. In the absence of any significant difference in response it is not surprising that the test of interaction between pressure and response shows no significance. Where both $R$ and $A$ are significant, $I$ provides a test of whether the competitive pressure exerted by the associate and the response to it of the indicator are additive in their effects on the character.

Two further statistical points remain to be made. Assuming additivity of the effects of pressure and response on the expression of the relevant characters, we can write $c_{T T}=\bar{c}+r+a ; c_{T L}=\bar{c}+r-a ; c_{L T}=\bar{c}-r+a$; and $c_{L L}=\bar{c}-r-a$, where $r$ and $a$ are the deviations from the mean $(\bar{c})$ ascribable 
to the effects of response and pressure. Just as $R=\frac{1}{4}\left(c_{T T}+c_{T L}-c_{L T}-c_{L L}\right)^{2}$ is the SS traceable to the difference between the two lines $T$ and $L$ in their responses to competitive pressure, so $r=\frac{1}{4}\left(c_{T T}+c_{T L}-c_{L T}-c_{L L}\right)$ and similarly $a=\frac{1}{4}\left(c_{T T}-c_{T L}+c_{L T}-c_{L L}\right)$. For character $p_{a}$ they become $r=$ $0 \cdot 04105 \pm 0.04305$ and $a=0 \cdot 12960 \pm 0 \cdot 04305$, which of course yield the same values of $t$ as we have already found in the analysis of variance. $R$ will be added to $\bar{c}$ when $T$ is the indicator and subtracted when $L$ is the indicator, and similarly $a$ will be added when the associate is $T$ and subtracted where it is $\mathrm{L}$. The difference between the two lines in response is $2 r$ and in pressure is $2 a$.

The second point to be noted is that a $t$ test was used in this analysis because the duplicate error variance of this experiment, and with it the error variance of the $c$ values derived from it, was based on only $12 \mathrm{df}$. Where the estimate of the basic error is based on $30 \mathrm{df}$ or more, a $\chi^{2}$ test of significance can be used, as was done by Mather $e t$ al. in their analysis of the data from Lolium perenne.

\section{Results}

\section{(i) Lolium perenne}

The competition experiment by Mather et al., with $L$. perenne involved three genotypes, B, C, and E. C and E were of similar phenotype, having large leaves and erect growth habits, and being less densely tillering. B on the other hand was short-leaved, densely tillering and showing a prostrate growth habit. C and $\mathrm{E}$ were derived from bred cultivars but $\mathrm{B}$ was from an indigenous population in South Wales. The character observed was mean weight in mgms of dry matter, transformed by taking its square root. Monoculture series were grown of all three genotypes, and all the three possible duoculture series, $B$ with $C, B$ with $E$ and $C$ with $E$, were also raised. Two randomised blocks were grown, each block including two examples of each monoculture and each duoculture, one of which was assigned to a regime of frequent $(F)$ and the other to a regime of infrequent (R) cutting.

The analysis followed the pattern described by Mather and Caligari, but was extended to include the derivation of the $c$ values from the variates $b_{m}$ and $b_{d}$ estimated from the data. Since all possible monocultures and duocultures were raised, all the nine $c$ values were obtained that are possible with three clones present as both indicators and associates, and they were obtained under both the $F$ and the $R$ cutting regimes. These are set out in Mather et al.'s table 7, a modified form of which is reproduced in our table 3. The error variance of the individual $c$ values was found to be 0.0001204 or 102.4 if we multiply by $10^{6}$ to put it on the same basis as the mean squares (MS) derived from table 3.

The $8 \mathrm{df}$ among the nine $c$ values were partitioned by Mather et al., into 2 for differences among the three genotypes as indicators (i.e., for differences in response), 2 for differences among them as associates (i.e., for differences in pressure or aggressiveness) and 4 for the $R \times A$ interaction (see their table 8 where it is shown as $I \times A$ ). This analysis of variance showed that (i) genotype $B$ differed markedly from $C$ and $E$ both in its response to competitive pressure and in the pressure that it exerted; (ii) $\mathrm{C}$ 
TABLE 3

Competition values (c) in the nine combinations of the three Lolium genotypes as indicator and associate, under the frequent $(\mathrm{F})$ and infrequent $(R)$ cutting regimes. The expected values are shown in italics below the observed values

\begin{tabular}{crrrlrrrr}
\hline & \multicolumn{3}{c}{ Frequent (F) } & \multicolumn{5}{c}{ Infrequent (R) } \\
& B & C & E & Mean & B & C & E & Mean \\
\hline Associate & & & & & & & & \\
B & 9.124 & 1.214 & 9.857 & 6.762 & 22.027 & 32.642 & 13.357 & 22.675 \\
& 4.950 & 8.347 & 6.988 & & 16.055 & 28.592 & 23.378 & \\
C & 28.071 & 56.961 & 44.357 & 42.905 & 55.571 & 102.786 & 95.786 & 84.714 \\
& 31.409 & 52.961 & 44.344 & & 59.982 & 106.820 & 87.341 & \\
E & 25.071 & 47.643 & 33.821 & 35.512 & 51.071 & 93.714 & 78.214 & 74.333 \\
& 25.997 & 43.835 & 36.703 & & 52.632 & 93.730 & 76.638 & \\
\hline \multirow{2}{*}{ Mean } & 20.785 & 35.048 & 29.345 & 28.393 & 42.890 & 76.381 & 62.452 & 60.574 \\
\hline
\end{tabular}

and $\mathrm{E}$ gave sub-significant differences in both response and pressure, but an analysis omitting $B$ gave evidence that there was a difference between them in the competitive pressures that they exerted, though there was no evidence of a difference in response; (iii) there was a significant $R \times A$ interaction in respect of the comparison between $B$ on the one hand and $C$ and $E$ taken together on the other; and (iv) there was a significant interaction between the treatment regime and the difference in pressure between $B$ on the one hand and $C$ and $E$ taken together on the other.

We can now take the analysis further. The significant interaction between the differences of $\mathrm{B}$ and $\mathrm{C}+\mathrm{E}$ in response and pressure shows that a simple additive model is not satisfactory for combining pressure and response to give the overall competition values. If, however, instead of finding the expectation on an additive basis as, for example, summing the means of the middle row and the middle column and then deducting the overall mean of the nine relevant $c$ values to obtain the expectation for $c_{C C}$ under the $F$ regime $(i . e .42 \cdot 905+35 \cdot 048-28 \cdot 393=49 \cdot 560)$, we multiply the marginal means and divide by the overall mean (for example $42.905 \times 35 \cdot 048 \div 28 \cdot 393=52.961$ ) to give a multiplicative expectation, the significant interaction between response and pressure vanishes. The expectations $(E)$ so obtained are given below the observed values $(O)$ in table 3. $S(O-E)^{2}=123.154$ for the $\mathrm{F}$ and 264.456 for the $\mathrm{R}$ regimes. Dividing by $V_{c}=102.4$, we find $\chi_{4}^{2}=1.203$ and $\chi_{4}^{2}=2.583$ for the two regimes. Not only is neither $\chi^{2}$ significant, but in neither case would it be significant even if we regarded it as attaching wholly to the single df, $R_{1} \times A_{1}$, which Mather $e t$ al. found to have a probability between 0.01 and 0.001 on the additive basis assumed in their analysis of variance. In these grasses the two components of competitive interaction, pressure and response, combine in a multiplicative fashion rather than additively.

With multiplicative relationships of this kind variation between the clones is to be measured by ratios rather than arithmetic differences. If we take as the yardstick genotype $\mathrm{C}$ which shows the strongest competitive interaction whether an associate or indicator, we find that $B$ and $E$ respectively show $62 \cdot 356 / 105 \cdot 143=0 \cdot 593$ and $88 \cdot 037 / 105 \cdot 143=0 \cdot 837$ the 
TABLE 4

The $\mathrm{a}$ and $\mathrm{r}$ values of the Lolium genotypes $B$ and $E$ relative to those of genotype $C$ under the two cutting regimes

\begin{tabular}{lcccccc}
\hline & $r_{\mathrm{B}}$ & $r_{\mathrm{C}}$ & $r_{\mathrm{E}}$ & $a_{\mathrm{B}}$ & $a_{\mathrm{C}}$ & $a_{\mathrm{E}}$ \\
\hline Frequent (F) & 0.593 & 1 & 0.837 & 0.158 & 1 & 0.828 \\
Infrequent (R) & 0.562 & 1 & 0.818 & 0.268 & 1 & 0.877 \\
\hline F/R & 1.055 & 1 & 1.023 & 0.590 & 1 & 0.944 \\
\hline
\end{tabular}

response of $C$ and exert 0.158 and 0.828 the competitive pressure under the $\mathrm{F}$ regime. These figures are collected together with the corresponding ratios from the $R$ regime in table 4. Despite the overall doubling of the yield of dry matter under the $R$ regime relative to $F$, the responses of $B$ and $E$ relative to $C$ remain virtually constant. Nor does the pressure exerted by $E$ relative to $C$ change materially with the regime; but $B$ is relatively a much stronger competitor under $\mathrm{R}$ than under $\mathrm{F}$. This difference is brought out even more strikingly if we look at the $F / R$ ratios at the bottom of the table. Although the response of $B$ relative to $C$ and $E$ is effectively unchanged by the alteration in cutting regime its relative pressure is not. Evidently competitive pressure is a more labile component of competition than is response. This can also be seen in a different way, which indeed emerges from the analysis of variance by Mather et al., where the MS variation among genotypes in respect of pressure exceeds that in respect of response by a factor of nearly 5 . The same emerges from a comparison of the ratios in table 4 which on pooling over regimes yield an MS in respect of pressure exceeding that in respect of response by a factor of nearly 4.

\section{(ii) Drosophila melanogaster}

The competing genotypes used in the three experiments whose results will now be analysed could not be distinguished by their chaeta numbers as were those of the T,L experiment, or indeed by any other obvious feature of the wild-type genotype. Marker genes had therefore to be used, $y^{2}$ in all three experiments and $w^{a}$ additionally in the third of them. The first two experiments were designed in such a way as also to test whether any effects on competitive values were associated with the,$+ y^{2}$ difference.

\section{(a) The A,C, experiment}

The two genotypes, $\mathrm{A}$ and $\mathrm{C}$ involved here were both $F_{1}$ s between pairs of inbred lines, $\mathrm{A}$ being $6 \mathrm{CL} \times$ Wellington (i.e., the $\mathrm{F}_{1}$ of the two lines in the experiment analysed in Section 2) and $C$ being Edinburgh $\times$ Samarkand. $y^{2}$ was introduced into all four of these parental inbreds, so permitting the production of a $y^{2}$ version of each $\mathrm{F}_{1}$. The $y^{2}$ version of $\mathbf{A}$ will be distinguished as $\mathrm{B}$, and that of $\mathrm{C}$ as $\mathrm{D}$. The reference density of eggs $(N)$ was 144 and $x$ took the values $0,-36,-72$ and -108 . Each culture vial was given $60 \mathrm{mgm}$ of yeast in $0.5 \mathrm{ml}$ of water. All four $F_{1} \mathrm{~s} \mathrm{~A}$, 
B, C and D were raised in monoculture series, and all the duoculture series in which the two constituents could be distinguished by body colour were raised too, viz., A with $\mathrm{B}, \mathrm{A}$ with $\mathrm{D}, \mathrm{C}$ with $\mathrm{B}$ and $\mathrm{C}$ with $\mathrm{D}$. Thus each of the four sets of results for analysis consisted of data from one monoculture and two duoculture series, e.g., A (as indicator) in monocultures and with $B$ and with $D$ in duocultures. The characters followed were the same as in the T,L experiment of Section 2: $p_{a}$, the proportion of eggs successfully developing into adults, expressed in angles; and $w$, the mean weight of the adults obtained. The complete experiment was carried out on three separate occasions, so providing the replication necessary for estimating the replicate error variation. Two cultures of each combination of flies were raised on each occasion and the mean of the pair used as the basic datum for that combination on that occasion.

Since there were ten combinations raised using each of the four $F_{1}$ 's as indicator on each occasion, there were in principle $10 \times 2$ df from each indicator for estimating the replicate error. Two of these were however sacrificed in correcting for overall differences between the three occasions, and the correction was made separately for each of the four indicators, thus leaving $18 \times 4=72 \mathrm{df}$ for the overall estimate of replicate error variation, which was found to be 9.51196 for $p_{a}$ and 0.0077707 for $w$. The values of $p_{a}$ and $w$ found for each combination in the experiments were averaged over the three occasions and these means used in the analysis. The error variation of such a mean of three observations is 3.17065 for $p_{a}$ and 0.0025902 for $w$.

The analysis of the 10 means obtained with each of the four indicators (i.e. 4 from the monoculture series and 3 from each of the two duoculture series) was carried out in the same way as that described by Mather $e t$ al., whose experiment indeed had the same structure as this one. Four parameters were estimated from the 10 observed mean values; $e$ (corresponding to $a$ in the two earlier papers) being the expression of the character at the reference density, $N$; together with three regression coefficients, $b_{m}$ from the monoculture series and $b_{s}$ and $b_{d}$ from the two duoculture series. This leaves $6 \mathrm{df}$ for testing the goodness of fit between the observed $(O)$ values and their expectations $(E)$ obtained using the estimates of the four parameters, by comparing $S(O-E)^{2}$ with the relevant error variation as found above. Pooling over the four indicator genotypes, $S(O-E)^{2}=72.4429$ based on $6 \times 4=24$ df for character $p_{a}$, yielding $\chi_{24}^{2}=22.85$ when divided by 3.1707 the relevant error variation, with $P=0 \cdot 7-0 \cdot 5$. Similarly the pooled $S(O-E)^{2}$ for $w$ was 0.031961707 , giving when divided by 0.0025902 , the relevant error variation, $\chi_{24}^{2}=12.34$ with $\boldsymbol{P}=0.98-0.95$. Clearly the model fits for both characters, with indeed a very high probability in respect of $w$. This was traceable to a particularly close fit of expectation and observation in the series where genotype $\mathrm{D}$ was the indicator.

The values of $e, b_{m}, b_{s}$ and $b_{d}$ from the four different indicator genotypes are collected together in table 5. Now, of the two duoculture regressions obtained with each indicator one, $b_{s}$, is from the duoculture where its associate had the same basic genotype differing only in respect of the marker gene $y^{2}$ (e.g., A with $\left.\mathrm{B}\right)$, while the other, $b_{d}$, is derived from the duoculture where the basic genotype as well as the marker gene was different (e.g., A with D). Thus $b_{s}$ is expected to have the value 0 , apart from any effect of $y^{2}$ itself, since each egg of the indicator genotype omitted 
COMPETITIVE INTERACTIONS

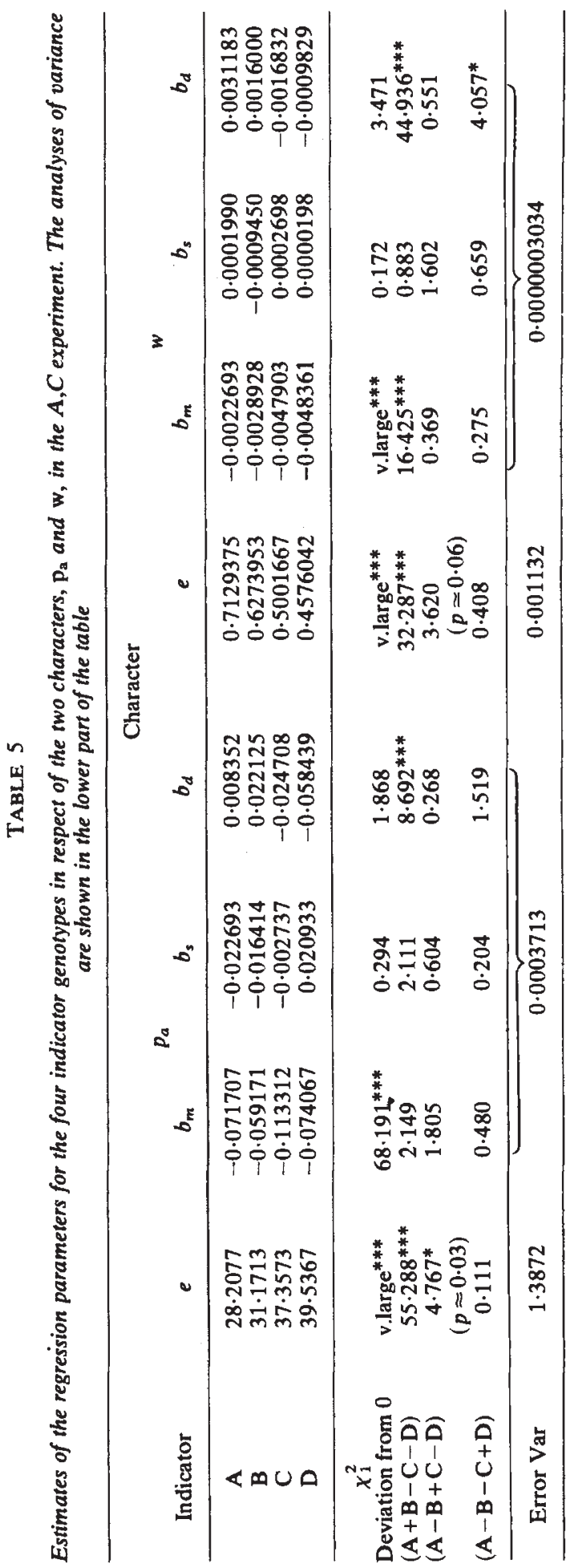


from the duoculture was replaced by another which differed only in the marker gene: it is in fact expected to be the $b_{o}$ of Mather and Caligari. $b_{d}$ on the other hand will show the effect of the difference in competitive ability between the two basic genotypes. Thus the four values of $b_{s}$, one from each of the four indicators, should differ from one another only by sampling variation. The analyses of variance in the lower part of table 5 shows that this is indeed the case.

Evidently of itself $y^{2}$ has a negligible effect on competitive value, and furthermore when introduced into the two basic genotypes it carried along no other genes materially affecting competitive value. True, $e$ was higher in respect of $p_{a}$ and lower in respect of $w$ in the two $y^{2}$ indicators, B and $\mathrm{D}$, than on their two wild-type counterparts, $\mathrm{A}$ and $\mathrm{C}$, the difference being just formally significant $(P=0.03)$ in the one case and just not so $(P=0.06)$ in the other. But $e$, being in the one case the proportion of eggs yielding adults and in the other the mean weight of the adults so yielded at a single density, $N$, depends on such factors as hatchability of the eggs and the ability of the developing individuals to utilise the available nutrients. It thus provides no direct evidence about competitive ability, which is measured by the rates of change of expression of the characters on density. No significant difference between $\mathrm{A}$ and $\mathrm{B}$ on the one hand or C and D on the other is to be seen in respect of any of the regression coefficients, $b$, on which the analysis of the competitive interactions is based. $y^{2}$ can therefore be treated as neutral in this analysis.

The $c$ values derived from the various $b$ 's are set out for both characters in table 6. The blanks represent combinations in which the two component genotypes could not have been separated by body colour and which were therefore not raised. Within each of the four groups separated by the dotted lines are $c$ values which in the absence of effect of the $y^{2}$ gene should differ only by sampling variation. The mean square differences within the groups were compared with the error variance of the individual $c$ values, found as $V_{c}=\frac{1}{2}\left(V_{b m}+V_{(b d-b m)}\right)=0.000360420$ for $p_{a}$ and 0.000000294442 for $w$. None of the mean square differences is significant when so tested except that for $c_{C B}-c_{D A}$ for character $p_{a}$ which gives $\chi_{1}^{2}=7 \cdot 388$ with $P=$ $0 \cdot 01-0.001$. This, however, is but one comparison out of 16 , i.e. 8 in $p_{a}$ and 8 in $w$. When the $\chi^{2}$ s are summed over all the 16 comparisons they yield $\chi_{16}^{2}=15 \cdot 470$ with $P \simeq 0 \cdot 5$. The $c$ values may thus be accepted as homogeneous within groups. The individual $c$ 's within each of the 8 groups ( 4 for each character) in table 6 have therefore been replaced by their means as shown in table 7 and we may proceed to estimate and test the aggressiveness $(a)$ and response $(r)$ values and interactions among them (i).

It should be noted, however, that for each character two of the four means are from four estimates of $c$ with the others coming from only two estimates. Thus the marginal differences of the table do not provide simple estimates of $a$ and $r$ as they did in the T,L experiment discussed in Section 2. The distributions of $a$ and $r$ are, in fact, partially correlated in the table as are the distributions of $i$ and $\bar{c}$, the overall mean value of $c$ in the experiment. We proceed, therefore, by a weighted least squares fitting of the four parameters $\bar{c}, r, a$ and $i$ using the model shown at the right of table 7. This yields the values shown in the lower part of the table. An "orthogonal" analysis of the kind used in Section 2 gives exactly the same values for these parameters, as indeed it must because the fitting of four 

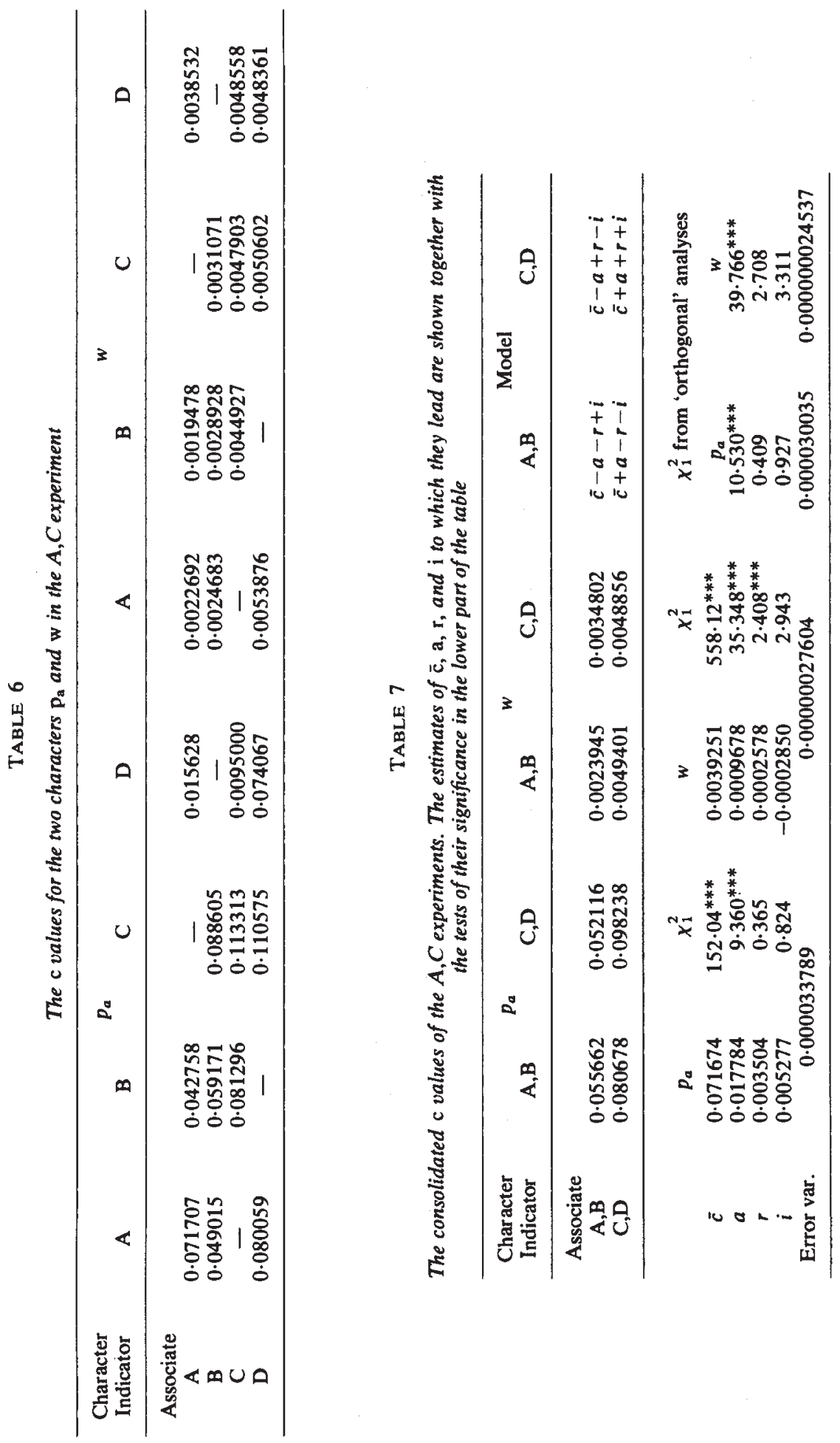
parameters to the four means results in a perfect fit. The partial correlations, however, affect the value of the error variance to which the estimates of the four parameters are subject. The least squares treatment shows it to be $V_{\bar{c}}=V_{a}=V_{r}=V_{i}=3 V_{c} / 32$, whereas if there were no correlations it would be $V_{c} / 12$. Analysis by "orthogonal" functions would thus spuriously reduce the error variance by $12 \frac{1}{2}$ per cent and so lead to a correspondingly over-high value for $\chi^{2}$ and over-estimate of significance.

The $\chi_{1}^{2} \mathrm{~s}$ testing the significance of the departures of the four parameters from $D$, are found as $\bar{c}^{2} / V_{\bar{c}}$ etc, and their values are shown alongside the estimates of the parameters in the lower part of the table. $\bar{c}$ is highly significant for both characters, which serves to confirm (if confirmation be necessary) that competition is operating. $a$ is also significant for both characters, but $r$ and $i$ are significant for neither of them. As in the T,L experiment the two genotypes, here $A(B)$ and $C(D)$, differ in the competitive pressures that they exert in respect of both characters; but there is no evidence that they differ in their responses to these pressures, and again not surprisingly there is no evidence of interaction between pressure and response. In conclusion we may note that were the analysis to have been carried out using "orthogonal" functions, just the same conclusions would have been reached; the use of the spuriously low error variance, though raising the $\chi_{1}^{2} s$ would not in this case have led to apparent significance of parameters $r$ and $i$ for either character.

\section{(b) The $C, H$ experiment}

This was of the same type as the A,C experiment just described. Two basically different $F_{1}$ genotypes were used, $C$ being, as before, the $F_{1}$ between the Edinburgh and Samarkand inbreds and $\mathrm{H}$ that between two inbreds, X27 and X28, derived from the Texas cage population. Again as before, $\mathrm{D}$ was $\mathrm{C}$ into which $y^{2}$ had been introduced as a marker gene, and I was similarly the $y^{2}$ version of $\mathrm{H}$. The same two characters, $p_{a}$ and $w$, were followed also. In principle, therefore, the analysis was just like that of the A,D experiment, though in the present experiment the reference density was set at 120 eggs per vial, $x$ took the values $0,-30,-60$ and -90 , and $45 \mathrm{mgm}$ of yeast were added to each culture vial. The complete experiment was carried out in duplicate.

This was an early experiment, however, and two complications arose. In the first place the reference density was set somewhat too high for the amount of food supplied, with the result that the yield of adults from the monocultures having 120 eggs per culture was both low and highly variable with the C,D genotype. The 120 egg monocultures of all genotypes were therefore omitted from the analysis, though the requirement was retained that all regression lines from the same indicator genotype pass through the same value, $e$, at the reference density. Secondly the experimental design was inadequate in that the monocultures and duocultures were not raised on the same occasions. This appears to have had no serious affect in the case of character $p_{a}$, but as we shall see complications arose with $w$, the mean weight of the adult flies obtained.

Apart from the omission of monocultures at the reference density, and the consequent adjustment to the $\mathbf{J}^{-1}$ matrix, the analysis followed the same lines as that of the $A, C$ experiment and so need not be described 
again in detail here. $y^{2}$ again proved to be neutral in its effect on competitive ability in respect of both characters: in particular all the values of $b_{s}$ failed to show any significant departure from 0 and the $c$ values proved to be homogeneous in each of the four groups into which the $c$ table is divisible. In the case of $p_{a}$ the observations gave an adequate fit with the model as tested by the comparison of $S(O-E)^{2}$ with the duplicate error variance. But with character $w$ the fit was unacceptably poor.

Inspection of the results showed that the difficulty with $w$ stemmed, at least in large part, from requiring the monoculture regression to pass through the same point, $e$, at the reference density as the duoculture regressions for the same indicator. Estimating $b_{m}$ separately by ordinary linear regression technique, but requiring $b_{s}$ and $b_{d}$ to share a common $e$ resulted in an adequate fit with the revised model and the further analysis of $w$ was carried out on this basis. A similar further analysis was also carried out in parallel using the unrevised model but taking the MS of $(O-E)$ as the basic error variance. In the case of $p_{a}$ the further analysis was of course carried out in the way used for the results of the A,C experiment.

Table 8 sets out the $c$ values for both $p_{a}$ and $w$ (using the revised model) in the same grouped form used for the A,C experiment in table 7. The estimates of the four parameters are set out in the lower part of the table; where they are also tested for significant departures from 0 . In the case of $w$ the results are similar to those obtained for both characters in the A,C experiment: $a$ departs significantly from 0 but $r$ and $i$ do not. There is thus good evidence of a difference between the two basic genotypes in respect of the competitive pressures they exert as associates, but no evidence of a difference in their responses to such pressures as indicators. Nor is there any evidence of interaction between pressure and response. We may note too that the parallel analysis using the unrevised model but with an estimate of error variance derived from $S(O-E)^{2}$ gave similar results and indeed a closely similar estimate $(0.001241)$ of $a$.

The other character, $p_{a}$, shows a quite different behaviour. Every parameter departs significantly from 0 , including the interaction parameter $i$. Thus $a$ and $r$ are clearly not additive. Nor does the multiplicative relationship of $a$ and $r$, which we inferred from the Lolium experiment, fit these results: indeed it gives a bigger $\chi^{2}$ for interaction than does the additive relationship when tested by $i$. Furthermore, when the indicator genotype is $C, D$ and the associate $H, I$, the $r$ value becomes significantly negative, so showing that far from exerting competitive pressure on $C, D$ and so reducing the chances of its eggs developing into adults, $\mathrm{H}, \mathrm{I}$ facilitates the development of $C, D$ and raises its chance of producing adults. This is also well shown by the plot of the regression lines obtained when the C,D basic genotype is the indicator (fig. 1 left). There is, however, no reciprocal effect when $\mathrm{H}, \mathrm{T}$ is the indicator and C,D in fact is, if anything, a hypercompetitor to $\mathrm{H}, \mathrm{I}$ (Fig. 1 right). Thus with these genotypes we can recognise an interactive relation between pressure and response.

\section{(c) The $X$ experiment}

Three inbred lines (X1, X5 and X15), all derived from the Texas cage population, were used in this experiment, $\mathrm{X} 1$ being wild-type but $\mathrm{X} 5$ and 


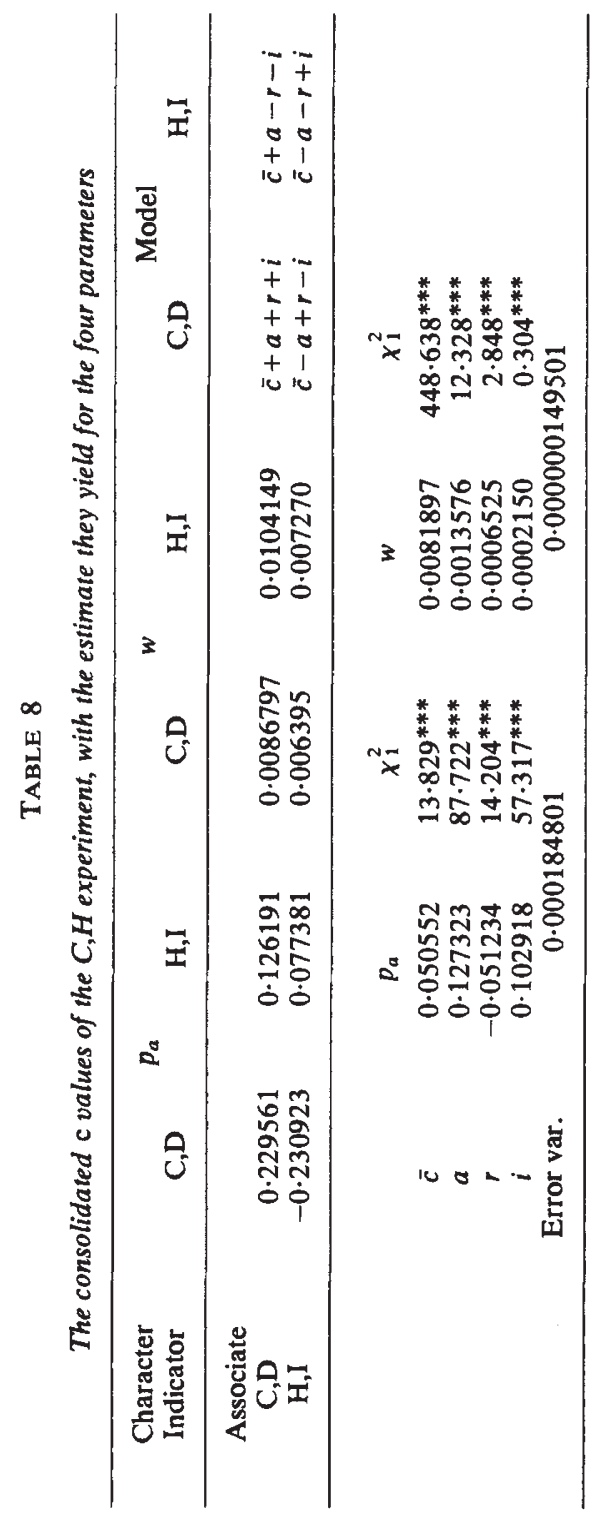




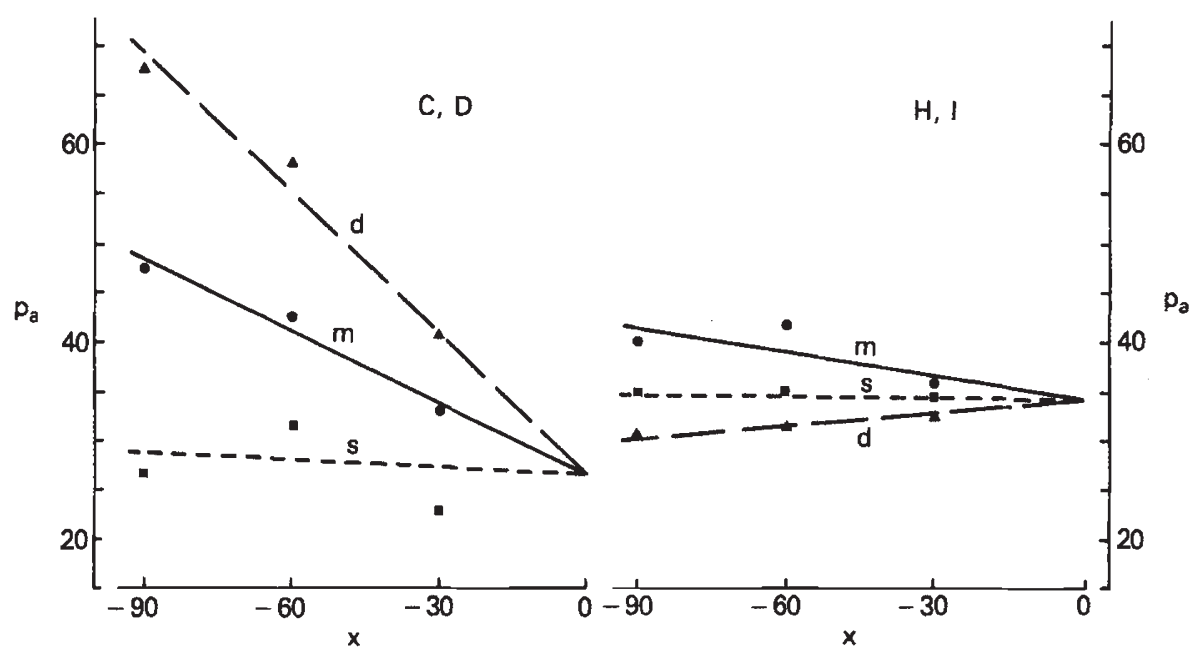

FIG. 1. Facilitation and competition for character $p_{a}$ in experiment $C, H$. The three regression lines, $m$ from monocultures, $s$ from duocultures with the marked strain of the same basic genotype, and $d$ from duocultures with the different basic genotype, are shown on the left for the series with $C, D$ as indicator, and on the right for the series with $H, I$ as indicator. In both cases $b_{s}$ agrees with its expected value 0 . With C,D as indicator $b_{d}$ is above $b_{m}$, so indicating facilitation of C,D individuals by $\mathrm{H}, \mathrm{I}$. With $\mathrm{H}, \mathrm{I}$ as indicator $b_{d}$ is below $b_{m}$ so indicating competition. The observed points, to which the lines are the best fitting regression, are shown by $\bigcirc \mathrm{for}, \square$ for $s$ and $\boldsymbol{\Delta}$ for $\mathrm{d}$.

$\mathrm{X} 15$ marked by the introduction of $y^{2}$ and $w^{a}$ respectively, so making possible the separation of adult flies into their respective lines in any mixture. We shall confine our attention to the behaviour of the three monocultures and the three possible duocultures, all of which were raised. Triocultures involving all three lines were also raised; but these are irrelevant to our present consideration. They will be analysed and discussed in a later report. The reference density, $N$, was 120 eggs per culture, $x$ took the values $0,-30,-60$ and -90 , and $45 \mathrm{mgm}$ of yeast were added to each culture vial. Only character $p_{a}$ was scored in this experiment, which was carried out in duplicate. The duplicate error variation, in the calculation of which a correction was made for overall difference between the duplicate series, was found to be $V=7 \cdot 348586$. The error variance of the $c$ values, derived from this $V$ turned out to be $V_{c}=0 \cdot 00115109$.

Since all the flies of line X1 were wild-type and those of X5 and X15 were $y^{2}$ and $w^{a}$ respectively, no tests of the effects of the marker genes were possible; but we have already seen that $y^{2}$ is neutral in its effect on competition and nothing in the present results suggests that $w^{a}$ produced any material disturbance either. The $c$ values derived from the three types of monoculture and the three types of duoculture are set out in table 9, in the lower part of which is the analysis of variance to which they give rise. There was of course no need with these results for a weighted least 
TABLE 9

The $\mathrm{c}$ values given by the three genotypes of the $X$ experiment together with the analysis of their variance

\begin{tabular}{|c|c|c|c|c|}
\hline Indicator & $\mathrm{X} 1$ & $\mathrm{X} 5$ & $\mathrm{X} 15$ & Mean \\
\hline \multicolumn{5}{|l|}{ Associate } \\
\hline $\mathrm{X} 1$ & 0.144003 & 0.068583 & 0.017857 & 0.076814 \\
\hline $\mathrm{X} 5$ & 0.311035 & 0.295898 & 0.125725 & 0.244219 \\
\hline $\mathrm{X} 15$ & $0 \cdot 180155$ & 0.119749 & 0.085608 & 0.128504 \\
\hline Mean & 0.211731 & $0 \cdot 161410$ & 0.076397 & $0 \cdot 149846$ \\
\hline Item & df & SS & MS & $x^{2}$ \\
\hline A & 2 & 0.044086 & 0.022043 & $38 \cdot 299^{* * *}$ \\
\hline $\mathbf{R}$ & 2 & 0.028075 & 0.014038 & $24 \cdot 390^{* * *}$ \\
\hline$A \times R$ & 4 & 0.005744 & 0.001436 & 4.990 \\
\hline TOTAL & 8 & 0.077905 & & \\
\hline$V_{c}$ & 46 & & 0.00115109 & \\
\hline
\end{tabular}

squares treatment of these results such as was necessary with $\mathrm{A}, \mathrm{C}$ and $\mathrm{C}, \mathrm{H}$ experiments.

It is clear from the analysis of variance that there are significant differences among both the competitive pressures, $a$, exerted by the three genotypes as associates, and the responses, $r$, to these pressures that they display as indicators. More detailed analysis leaves no doubt that all three lines differ from one another in both of these respects. The interaction item, $A \times R$, is not significant: indeed if the whole of the $\chi^{2}$ came from only interaction comparisons it still would not be significant. The $c$ values thus accord satisfactorily with the simple additive model $c=\bar{c}+a+r$ for their composition in terms of pressure and response. At the same time the $c$ values do not exclude a multiplicative relation of the type found in the Lolium experiment, but the additive model will be assumed as leading more easily to further analysis.

Values of $a$ and $r$ can be found by deducting the overall mean, $\bar{c}=$ 0.149846 from the line mean in the right hand margin of the table to give $a$ and from that in the lower margin to give $r$. These values are set out in table 10 . They are of course subject to the constraints that $S(a)$ and $S(r)$ must both equal 0 . The analysis of variance in table 9 shows that genotypes of Drosophila can differ in their responses to competitive pressure as well as in the pressures they exert. Table 10 shows us further that in respect of pressure, as measured by $a$, the three lines fall into the order X5>X15> $\mathrm{X} 1$, but in respect of response as measured by $r$ their order is X1>X $5>$ $\mathrm{X} 15$. Thus a higher ability to exert competitive pressure is not always found with a higher response to such pressure. Neither is the opposite always the case.

\section{Discussion}

These results reveal a variety of relationships between $a$, the competitive pressure that a genotype can exert on its fellows, and $r$, the response of a 
TABLE 10

The a and $\mathrm{r}$ values of the three genotypes in the $X$ experiment, expressed as deviation from the mean $c$ value of the experiment $(0 \cdot 143998)$

\begin{tabular}{cccc}
\hline Line & $\mathrm{X} 1$ & $\mathrm{X5}$ & $\mathrm{X} 15$ \\
\hline$a$ & -0.073032 & 0.094373 & -0.021342 \\
$r$ & $\mathbf{0 . 0 6 1 8 8 5}$ & 0.011564 & -0.073449 \\
\hline
\end{tabular}

$\bar{c}=0 \cdot 149846$

genotype to the competitive pressures under which it finds itself. Most of the information is supplied by the Drosophila experiments. The character $w$ (mean weight of adult flies) shows the same relationship in all the three experiments in which it was followed: a difference was found in the competitive pressures exerted by the two competing genotypes of each experiment but no evidence was obtained of any difference in the responses of the two to these pressures. Not surprisingly there is no evidence either of interaction between $a$ and $r$. The other character $p_{a}$ (proportion of eggs successfully developing into adult flies) also gives the same result in the T,L and A,C experiments. Clearly $a$ can vary without $r$ doing so, but no case was found of $r$ varying without $a$ doing so.

The $\mathrm{X}$ experiment, in which $p_{a}$ was the only character to be followed, shows however that both pressure and response can vary at the same time, though it provides no indication that they do so in a correlated fashion (table 10). We can take the analysis further. Noting that 0.0944 does not differ significantly from 0.0619 and that -0.0213 and -0.0115 do not differ significantly from 0 , and taking as the unit value 0.075 (which is close to the mean of the four unit values in the table) we can rewrite table 10 as:

\begin{tabular}{rrrr}
\hline & $\mathrm{X} 1$ & $\mathrm{X} 5$ & $\mathrm{X} 15$ \\
\hline$a$ & -1 & 1 & 0 \\
$r$ & 1 & 0 & -1 \\
\hline
\end{tabular}

Thus from at least this experiment there is a clear suggestion of two separable genetic systems operating in respect of character $p_{a}$, one governing variation in the competitive pressure and the other governing variation in response to that pressure, and each able to take values of some $0.075,0$ and -0.075 in deviation from a mean of about 0.14 in experiments carried out under conditions comparable to those that we used. Further discussions must however await a fuller genetical analysis of the determination of differences in pressure and response.

The $\mathrm{C}, \mathrm{H}$ experiment, though sharing with other experiments the simple relation $a \neq 0, r=0$ in respect of character $w$, shows a quite different relation between pressure and response in respect of character $p_{a}$. Here $a$ and $r$ show a highly significant interaction, $i$ lying between $a$ and $r$ in value. 
Furthermore, in duoculture, the presence of the $\mathrm{H}$ genotype raises the probability of a $\mathrm{C}$ type egg giving rise to an adult fly (fig. 1). Far from competing with individuals of genotype $\mathrm{C}$ those of genotype $\mathrm{H}$ appear to facilitate their successful development, though in the same duocultures $\mathrm{C}$ competes successfully with $\mathrm{H}$ : indeed one must assume that it does so the more successfully because of the additional $\mathrm{C}$ individuals whose development has been facilitated by the presence of $\mathrm{H}$ itself. $\mathrm{C}$ benefits from the presence of $\mathrm{H}$, though $\mathrm{H}$ suffers from the presence of $\mathrm{C}$; or to put it in terms of our notation $c_{H C}$ is positive in the familiar way, while $c_{C H}$ is negative.

Such an asymmetrical interaction could arise from a relationship like that described by Haj-Ahmad and Hickey (1982). Adult flies homozygous for the gene Amy ${ }^{\text {null }}$ cannot make the enzyme amylase for themselves and so die on a starch medium. They survive however if adults carrying Amy ${ }^{1}$, which can make amylase, are also present, because they obtain the product of amylase action from the secretions of the Amy ${ }^{1}$ individuals. In the present case, of course, any transfer between the individuals of the two genotypes must have been between larvae via the medium at an earlier stage, but this would appear to be a not unlikely possibility. There are products of the Amy locus other than Amy ${ }^{\text {null }}$ and Amy ${ }^{1}$ which vary in their activity, and so could account for the successful development of some flies in monoculture while developing in greater proportion in duocultures carrying a more efficient allele (De Jong and Scharloo, 1975). We do not know, however, that amylase is involved in the present case of facilitation, but a similar interaction might take place between genotypes differing in their abilities to produce other substances essential for development and survival on the medium we used, while at the same time being similarly capable of passing between the genotypes by excretion or secretion into the medium.

It is also relevant to note here that competitive interactions can be produced by such secretion or excretion into the medium by individuals of one genotype, of substances deleterious to other genotypes (Weisbrot, 1966). Huang et al. (1971) have indeed shown that a polymorphism for alleles at the esterase- 6 locus can be maintained in this way and Kojima and Huang (1972) further established that the inter-relation was densitydependent as competitive interactions obviously must be. Thus transfer of substances from individuals of one type to those of another can bring about both competitive and facilitative effects, though obviously this is not the only way in which facilitation, any more than competition, can arise.

The results from the rye-grass experiment differ from those from Drosophila in two striking ways. In the first place they contrast with those from the $\mathrm{X}$ experiment in that the three rye-grass genotypes gave $a$ and $r$, values which are positively correlated: $B$ has much the lowest values for both $a$ and $r, \mathrm{C}$ has the highest for the two parameters, and $\mathrm{E}$ is intermediate for both though much nearer to $C$ than to $B$. This is not surprising when we bear in mind that the mechanisms of competition must be very different in green plants which are sessile and flies which are mobile. Rye-grass plants of genotype B are short leaved, densely tillering and prostrate in habit, by contrast with their fellows of $C$ and $E$ which have larger leaves, fewer tillers and erect growth habits. A B plant will inevitably be overshadowed by its neighbours where they are $\mathrm{C}$ and $\mathrm{E}$ and it cannot move 
away from its unfortunate position as a fly larva can. It will be deprived of light and its food supply will thereby be reduced. As C and E grow they will put increasing pressure on $B$ which in turn will grow even less vigorously and any pressure it could exert initially on $C$ and $E$ will decline. So must its response to their pressure, since the weaker its growth the less response it can display. Thus we might expect the $a$ and $r$ values to be correlated. We would also expect the competitive advantages of $\mathrm{C}$ and $\mathrm{E}$ over $\mathrm{B}$ to be greater when the plants are rarely cut, since this will allow $C$ and $E$ to take full advantage of their erect habit in overshadowing $\mathrm{B}$; and the competitive advantages of $\mathrm{C}$ and $\mathrm{E}$ over $\mathrm{B}$ was indeed greater under the $R$ regime than under the $F$ cutting treatment.

The second way in which the results from Lolium stand in contrast to those from its counterpart three-genotype X experiment with Drosophila lies in the way that $a$ and $r$ appear to combine in a multiplicative way which departs significantly from the additive relationship which affords a perfectly satisfactory model for the Drosophila results. We do not of course know whether a similar contrast would be shown by competitive relations in other plants and other animals, or even by other genotypes of Lolium perenne and of Drosophila melanogaster. There is, however, some indication that the interaction between $a$ and $r$ in the Lolium experiment may arise from, or at least be exaggerated by, the extreme difference in habit between genotype $B$ on the one hand and $C$ and $E$ on the other, in that it is largely traceable to the values found for $c_{B B}$ measuring competition in monocultures of B. Though still low by comparison with $c_{C C}$ and $c_{E E}$, they are markedly higher than might be expected from those of $c_{B C}$ and $c_{B E}$, and particularly from those of $c_{C B}$ and $c_{E B}$. In other words $B$ is relatively a much better competitor with itself than might be expected from its performance in competition with $\mathrm{C}$ and $\mathrm{E}$, especially when looked at in terms of the competitive pressure it exerts as opposed to the response it displays. Like the values of $a$ and $r$ themselves, the way they combine to give the overall competitive value must refiect the basic biological properties of the competing organisms.

Acknowledgements. We gratefully acknowledge financial support from the Agricultural Research Council during the early part of this work. We are also grateful to Drs A. J. Birley and M. J. Lawrence for reading and commenting on the original manuscript.

\section{REFERENCES}

BREESE, E. L. AND HILL, J. 1973. Regression analysis of interactions between competing species. Heredity, 31, 181-200.

DE JONG, G. AND SCHARLOO, W. 1975. Environmental determination of selective significance or neutrality of amylase variants in Drosophila melanogaster. Genetics, 84, 77-94.

HAJ-AHMAD, Y. AND HICKEY, D. A. 1982. A molecular explanation of frequency-dependent selection in Drosophila. Nature Lond. 299, 350-352.

HUANG, S. L., SINGH, M. AND KOJIMA, K. 1971. A study of frequency-dependent selection observed in the esterase- 6 locus of Drosophila melanogaster using a conditioned media method. Genetics, 68, 97-104.

KOJIMA, K. AND HUANG, S. L. 1972. Effects of population density on the frequency-dependent selection in the esterase-6 locus of Drosophila melanogaster. Evolution, 26, 313-321. 
MATHER, K. AND CALIGARI, P. D. S. 1981. Measurement of competition. Heredity, 46, 239-254.

MATHER, K., HILL, J. AND CAligARI, P. D. S. 1982. Analysis of competitive ability among genotypes of perennial ryegrass. Heredity, 48, 421-434.

WEISBROT, D. R. 1966. Genotypic interactions among competing strains and species of Drosophila. Genetics, 53, 427-435. 